\title{
VHE Gamma-ray sources in view of the Cherenkov Telescope Array - A Review
}

\author{
Juan Cortina* \\ Institut de Fisica d'Altes Energies, Cerdanyola del Valles E-08193 Spain \\ E-mail: cortina@ifae.es
}

\section{for the CTA Consortium}

Imaging Atmospheric Cherenkov Telescopes (IACTs) have opened a new astronomical window at photon energies exceeding tens of $\mathrm{GeV}$ during the last decades. The technique relies on the detection of Cherenkov light produced by electromagnetic showers in the Earth's atmosphere using telescope mirrors with diameters of a few to tens of meters. A world-wide community is behind the design and construction of the next generation Cherenkov Telescope Array (CTA), consisting of two arrays of IACTs at the northern and southern hemispheres. I will review the astrophysics that IACTs are revealing at these energies, cover the design characteristics of CTA, the status of the project and the physics prospects of the instrument.

Frontier Research in Astrophysics - II

23-28 May 2016

Mondello (Palermo), Italy

\footnotetext{
* Speaker.
} 


\section{Instrumentation for Very High Energy $\gamma$-ray astrophysics}

Very High Energy (VHE; $>100 \mathrm{GeV}) \gamma$-rays are detected using space-based or ground-based detectors. From space Fermi-LAT[1] is performing the deepest survey to date of the $\gamma$-ray sky from $20 \mathrm{MeV}$ up to energies in excess of $100 \mathrm{GeV}$, although with limited sensitivity above $10 \mathrm{GeV}$ due to its relatively small collection area $\left(0.8 \mathrm{~m}^{2}\right)$.

VHE $\gamma$-rays are also detected from the ground. When they enter the Earth's atmosphere they develop a shower of lower energy $\gamma$-ray, electrons and positrons. Imaging Atmospheric Cherenkov Telescopes (IACTs) rely on the detection of Cherenkov light produced by this shower in the atmosphere and collected in telescope mirrors with diameters of a few to tens of meters. The light is detected using arrays of hundreds to thousands of photodetectors such as Photomultipliers or Geiger-mode Avalanche PhotoDiodes (G-APDs aka Silicon Photomultipliers or SiPM). The timing and the distribution of charge in the camera for each individual event can be used to discriminate primary $\gamma$-rays from the background of light of night sky and cosmic ray showers. Observing the same shower with several telescopes located at distances of $\sim 100 \mathrm{~m}$ allows further background rejection and a significant improvement in angular and spectral resolution.

The IACT technique was pionered by the Whipple telescope in 1989. The HEGRA array introduced stereoscopy during the last years of the 20th century. The MAGIC[2, 3], H.E.S.S.[4] and VERITAS[5] arrays have brought the technique to maturity during the last 15 years by discovering almost 200 VHE sources ${ }^{1}$. For a historial review of the evolution of the field the reader is referred to [6]. Most importantly the latest generation of IACTs has established that several populations of astronomical objects are active at VHE, effectively opening a new astronomical window.

Also from the ground air shower instruments such as Milagro[7], Tibet[8] and HAWC[9] detect $\gamma$-rays at higher energies (above some hundreds of $\mathrm{GeV}$ to typically $\mathrm{TeV}$ ), a comparable collection area of $\sim 80000 \mathrm{~m}^{2}$, but with a much larger FOV of $\sim 5000 \mathrm{sq}$ deg and higher duty cycle. They are non-tracking instruments. Unfortunately they are not as efficient as IACTs in eliminating the cosmic ray background, so they suffer from a worse sensitivity and they have much worse angular or spectral resolutions.

\section{The Cherenkov Telescope Array}

A world-wide community is behind the design and construction of the next generation IACT array: the Cherenkov Telescope Array (CTA[10]).

CTA aims at enhancing the flux sensitivity of current IACTs by as much as a factor 10 in the core energy domain (approaching the $1 \mathrm{mCrab}$ level for 50 hour integration), achieving an angular resolution as low as $0.1^{\circ}-0.05^{\circ}$ between $0.1-1 \mathrm{TeV}$ and extending the energy coverage to the range from $20 \mathrm{GeV}$ to at least $300 \mathrm{TeV}$. In doing so CTA needs to combine telescopes of different mirror sizes and to build up to a hundred of them.

CTA also aims at providing full-sky coverage. It will consist of two sites, one in the Northern Hemisphere (CTA-North) and one in the South (CTA-South).

The very wide energy range covered by CTA-South necessitates the use of at least three different telescope types: Large Size Telescopes (LSTs), Medium Size Telescopes (MSTs) and Small

\footnotetext{
${ }^{1}$ For an up to date list of VHE sources visit http://tevcat.uchicago.edu/
} 
Size Telescopes (SSTs). In CTA-North, the scientific focus will be on extragalactic science and hence (due to gamma-gamma absorption on Mpc scales) only limited sensitivity is required much beyond $10 \mathrm{TeV}$. CTA-North can therefore be implemented with only LSTs and MSTs.

\subsection{Description of the telescopes}

\subsubsection{LSTs}

LSTs[11] allow to extend the energy range of CTA to the lowest energies down to $20 \mathrm{GeV}$. Currently the CTA baseline arrays feature 8 LSTs, 4 at CTA-North and 4 more at CTA-South.

Each LST is equipped with a primary tessellated mirror dish of $23 \mathrm{~m}$ diameter, supported by a structure made mainly of carbon fibre reinforced plastic tubes and aluminum joints. This solution guarantees light weight (around 100 tons), essential for fast repositioning to any position in the sky in $<20$ seconds.

The camera of the LST shares many elements with the NectarCam proposed for use in the MSTs. With a weight of less than 2 tonnes the camera is comprised of 265 PMT modules that are easy to access and maintain. Each module has 7 channels, providing the camera with a total of 1855 channels. Hamamatsu photomultiplier tubes with a peak quantum efficiency of 42\% (R11920-100) are used as photosensors. All control, readout based on the DRS4 (Domino Ring Sampler version 4) chip and custom trigger electronics are embedded in the camera.

The design is now complete and production of the first LST, which will serve as a prototype for the remaining seven, is well underway. In 2016 the first LST will be installed at the Roque de los Muchachos Observatory on the Canary island of La Palma (Spain). The goal is to produce all the LSTs over a period of six years, starting with the installation of the first LST by the end of 2016 and ending with the commissioning of the last LST in 2021.

\subsubsection{MSTs}

The sensitivity in the energy range between $100 \mathrm{GeV}$ and $10 \mathrm{TeV}$ will be dominated by up to 40 MSTs[12] distributed over both observatory sites. In CTA's baseline design 25 MSTs shall be deployed on CTA-South and 15 MSTs on CTA-North.

An extension for the southern observatory with medium size telescopes with a dual-mirror design (Schwarzschild-Couder Telescope, SCT[13]) and an innovative camera based on SiPM is planned.

The MST is a modified Davies-Cotton telescope with a reflector diameter of $12 \mathrm{~m}$ on a polar mount, and a focal length of $16 \mathrm{~m}$. Two camera concepts are considered for the MST. They are dubbed NectarCam[14] and FlashCam[15]. The camera Field of View is larger than $7^{\circ}$.

The mirrors for the MSTs are realised as spherical facets of hexagonal shape (1.2 m flat-toflat). Specular reflectivity of the reflector at all wavelengths from $300-550 \mathrm{~nm}$ is $>85 \%$. Optical PSF for $80 \%$ light containment over $80 \%$ of the camera FoV is $<0.18^{\circ}$. Three different designs from France, Italy and Poland are tested to prepare a cost-effective solution that allows massproduction matching the CTA schedule.

A prototype for the mechanics, the drive assembly and auxiliary components of the MST was built in 2012 in Berlin. The goals of the prototype were to test and optimise the behaviour 
of mechanical structure under loads and environmental influences, the behaviour of the drive and safety concepts and the functionality of the mirror control and alignment.

\subsubsection{SSTs}

The SSTs[16] will cover the high end of the CTA energy range, between a few $\mathrm{TeV}$ and 300 $\mathrm{TeV}$. About 70 SSTs are needed in order to meet CTA's sensitivity requirements. The goal lightcollection area of the SST sub-array above $100 \mathrm{TeV}$ is over $7 \mathrm{~km}^{2}$. Monte Carlo studies indicate that this can be achieved with an array of about 70 telescopes at separations of about $250 \mathrm{~m}$ with 4 m diameter mirrors. At $10 \mathrm{TeV}$, the angular resolution must be at least $0.04^{\circ}$ and $0.03^{\circ}$ at $100 \mathrm{TeV}$. Another important requirement for the SSTs is the field of view (FoV), which needs to be $>8^{\circ}$.

Three different SST implementations are being prototyped and tested: one single-mirror DaviesCotton design (SST-1M) and two dual-mirror Schwarzschild-Couder optics designs (SST-2M ASTRI and SST-2M GCT). The current expectation is that the array will include 20 SST-1M telescopes, 35 ASTRI SST-2M telescopes[17] and a similar number of SST-2M GCT telescopes.

The dual-mirror designs allow excellent imaging across a wide field of view with a short focal length. The resulting small plate scale allows the use of SiPMs, assembled in very compact cameras. The ASTRI camera is based on SiPMs, while the GCT is considering the use of SiPMs or multi-anode PMTs. The SST-1M also uses silicon sensors, in the form of individual hexagonal pixels equipped with light concentrators (Winston cones).

A prototype of an ASTRI telescope was installed in September 2014 in Mount Etna, Sicily. A prototype of the GCT recorded CTA's first ever Cherenkov light while undergoing testing on November 2015 in Meudon, France. A prototype of the SST-1M should be deployed in 2016. 

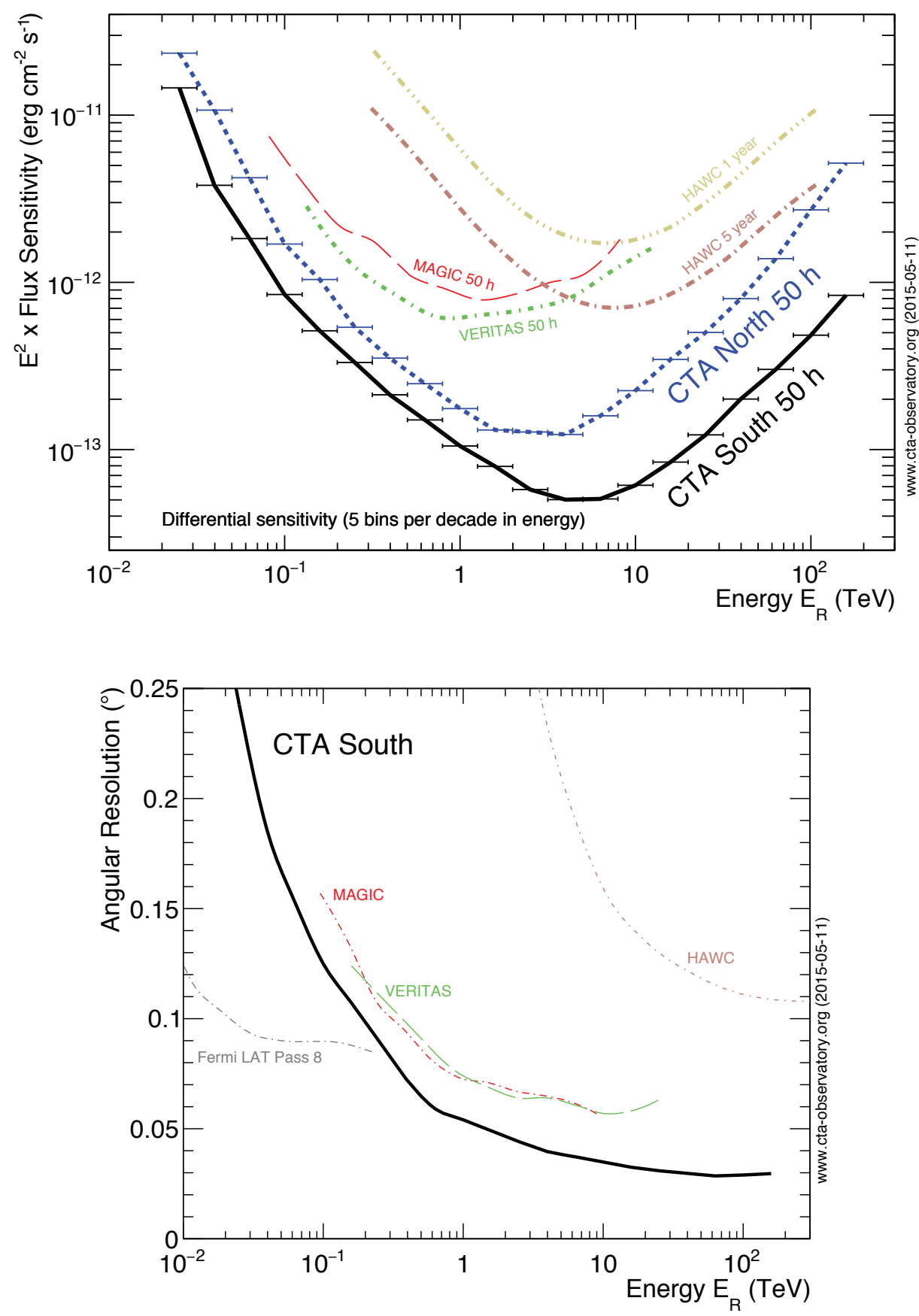

Figure 1: Comparisons of the performance of CTA with selected existing gamma-ray instruments. Top: differential flux sensitivity for five independent five standard deviation detections per decade in energy. Additional criteria applied are to require at least ten detected gamma-rays per energy bin and a signal/noise ratio of at least 20. Bottom: angular resolution expressed as the $80 \%$ containment radius of reconstructed gamma rays (the resolution for CTA North is similar). Figure from [19]. 


\subsection{Expected performance}

The large number of telescopes $(\sim 100)$ and wide fields of view of each individual telescope $\left(>8^{\circ}\right)$ for most of the energy range result in a CTA collection area which is one or more orders of magnitude larger than current generation IACTs at essentially all energies. This is beneficial for imaging, spectroscopy and the generation of light curves. A collection area in excess of 1 $\mathrm{km}^{2}$ is essential at the highest energies where there is essentially zero background even in long exposures and sensitivity is limited by the collection of sufficient signal photons. For very short time scale phenomena (see below), CTA is background free over much of its energy range and the large collection area is the key performance driver. At the lowest energies the largest telescope mirrors allow overlap with satellite-based detectors. The wide field of view allows to measure very large objects and diffuse emission, and it makes CTA into a very efficient instrument for large scale surveys.

Figure 1 compares the sensitivity and angular resolution of the CTA arrays to a selection of existing gamma-ray detectors. The improvement in the point-source sensitivity of CTA with respect to current instruments is a consequence of the combination of improved background rejection power, increased collection area and improved angular resolution. The improved background rejection power is achieved primarily through high image multiplicity and is particularly important for the study of extended, low-surface brightness objects and for low-flux objects where deep exposures are required.

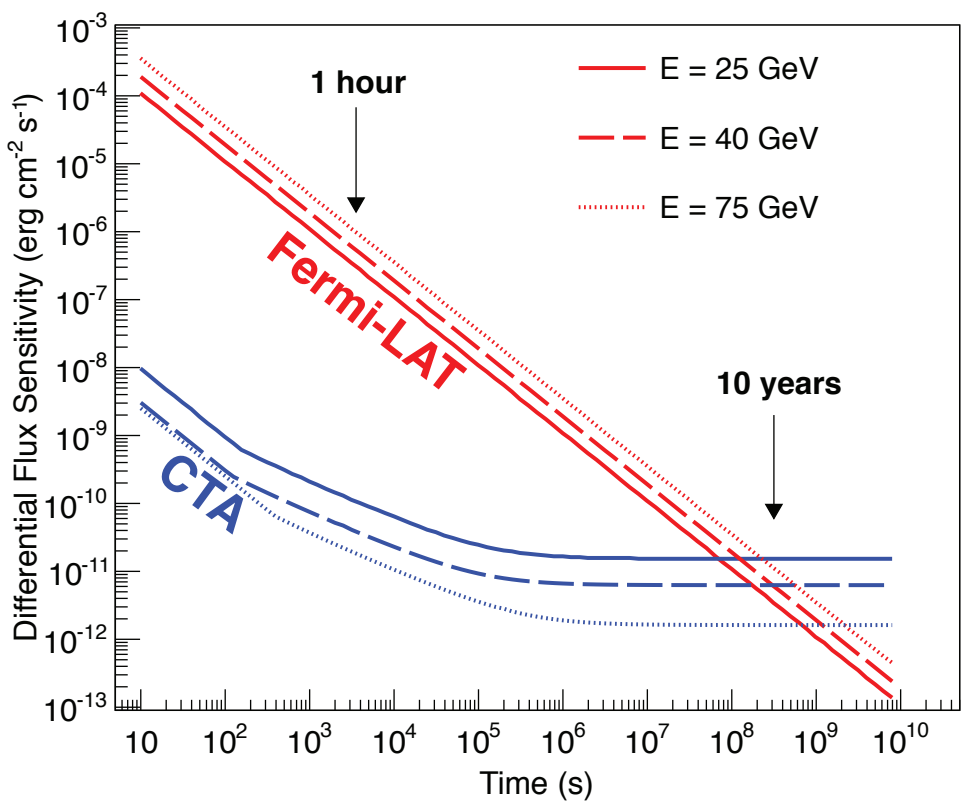

Figure 2: Comparison of the sensitivity of CTA and Fermi-LAT in the energy range of overlap versus observation timescale. Differential flux sensitivity at three energies is compared. Figure from [19].

CTA is a uniquely powerful instrument for the exploration of the violent and variable universe. It has unprecedented potential both in terms of energy reach and sensitivity to short timescale phenomena. Figure 2 compares the sensitivity of CTA to that of Fermi-LAT as a function of observa- 
tion time. CTA has four orders of magnitude better sensitivity to minute timescale phenomena at around $30 \mathrm{GeV}$. Even at variability timescales of 1 month, CTA will be a factor 100 more sensitive than Fermi-LAT, and only for emission which is stable over the full mission lifetime of Fermi are the sensitivities of the two instruments comparable in the lowest part of the CTA energy range. Instruments such as HAWC, which have sensitivity in the higher part of the CTA range, are also limited at short timescales by (relative to CTA) small collection areas, as well as low signal to noise and limited precision.

\subsection{Status of the project}

CTA is included in the 2008 roadmap of the European Strategy Forum on Research Infrastructures (ESFRI). It is one of the "Magnificent Seven" of the European strategy for astroparticle physics published by ASPERA, and highly ranked in the "strategic plan for European astronomy" (leaflet) of ASTRONET. In addition CTA is a recommended project for the next decade in the US National Academies of Sciences Decadal Review.

The instrument is ready for production. A Technical Design Report for the instrument was completed last year and passed a Critical Design Review in June 2015. The CTA consortium expects to secure funding for the project in 2016. Provided that funding is available on time the full array may be deployed in 2024.

After a long process to select the best sites of the northern and southern arrays CTA decided in 2015 to enter into detailed contract negotiations to host them at European Southern Observatory Paranal grounds in Chile and at the Roque de los Muchachos Observatory in La Palma, Spain. Array site negotiations are expected to finish this year. After site preparation the installation of the first telescopes should take place in 2017.

On June 2016, the governing body of CTA, the CTA Council, selected Bologna as the host site of the CTA Headquarters and Berlin - Zeuthen for the Science Data Management Centre.

\subsection{Observation planning}

An essential feature of CTA is that, contrary to previous IACTs, it will be the first groundbased gamma-ray open observatory, meaning that CTA will work essentially on the basis of the submission of observation proposals and data will be opened to the entire astronomical community.

During the first years however a significant fraction of the observing time will remain proprietary to the constructing consortium. The consortium has prepared a proposal for use of 40-50\% of the available time in the first ten years of operation: a Core Programme of highly motivated observations which will provide legacy data products and benefit future users of the observatory, as well as the entire astronomical community. The programme is made up of individual Key Science Projects (KSPs). A full description of the KSPs can be found in [19]. The KSPs have been prepared over several years by the CTA Consortium, with community input gathered via a series of workshops connecting CTA to neighbouring communities. A major element of the programme is the search for dark matter, in particular the annihilation signature of WIMPs. The strategy for dark matter detection places the expected cross-section for a thermal relic within reach for a very wide range of WIMP masses from around $200 \mathrm{GeV}$ to $20 \mathrm{TeV}$, making CTA extremely complementary to other approaches. CTA will also conduct a census of particle acceleration in the universe, with 
quarter-sky extragalactic, full-plane Galactic and Large Magellanic Cloud surveys planned. Additional KSPs are focused on transients, acceleration up to PeV energies in our own galaxy, active galaxies, star-forming systems on a wide range of scales, and the Perseus cluster of galaxies. Highlevel data products will be provided and will benefit a wide community. Together they will provide a long-lasting legacy for CTA.

\section{Physics at VHE - A look into the CTA future}

In a nutshell the scientific case of current IACTs and CTA can be summarized with the following questions:

- Theme 1: Understanding the Origin and Role of Relativistic Cosmic Particles

- What are the sites of high-energy particle acceleration in the universe?

- What are the mechanisms for cosmic particle acceleration?

- What role do accelerated particles play in feedback on star formation and galaxy evolution?

- Theme 2: Probing Extreme Environments

- What physical processes are at work close to neutron stars and black holes?

- What are the characteristics of relativistic jets, winds and explosions?

- How intense are radiation fields and magnetic fields in cosmic voids, and how do these evolve over cosmic time?

- Theme 3: Exploring Frontiers in Physics

- What is the nature of Dark Matter? How is it distributed?

- Are there quantum gravitational effects on photon propagation?

- Do axion-like particles exist?

In what follows I will briefly review the results of the previous generation of IACTs and touch on just a few of the improvements that we expect from CTA.

\subsection{Galactic physics}

\subsubsection{Supernova Remnants, PeVatrons}

Supernova remnants (SNRs) are able to provide enough energy to explain the population of galactic cosmic rays up to the so-called "Knee" of the spectrum, at around $1 \mathrm{PeV}$, if only $10 \%$ of the supernova kinetic energy is tranfered to accelerated particles. Particles are accelerated via diffusive shock acceleration at the expanding SNR shocks[20]. The acceleration of cosmic rays at SNR shocks is accompanied by an amplification of the magnetic field that can boost the acceleration of protons up to Knee energies. Nevertheless this amplification mechanism is not completely understood, and thus it is still unclear whether or not SNRs can act as cosmic-ray "PeVatrons". In 
other words, one hundred years after their discovery, the question of the origin of cosmic rays is still open.

Seven shell type SNRs are currently identified as TeV emitters (see [21] and references within): Cas A, Tycho, SN 1006, RX J1713.7-3946, RX J0852-4622, RCW 86, and G353.6-0.7. These results imply effective production of relativistic particles. These particles may be hadrons and the corresponding particle spectra would extend to $100 \mathrm{TeV}$, with a corresponding energy of $10^{50} \mathrm{erg}$. This would be consistent with the paradigm of cosmic ray acceleration in SNR. However in many cases $\gamma$-rays may be also be produced by the Inverse Compton (IC) emission of leptons.

On the other hand the $\gamma$-ray "echo" of the faded out accelerators can still be detected, even ten thousand years after the acceleration phase. After escaping the shell cosmic rays interact with the surrounding atomic and molecular gas and produce $\gamma$-rays with a spectrum that is different than the spectrum of the SNR and the galactic diffuse emission. What is more, $\gamma$-ray emission is significantly enhanced if escaping cosmic rays find massive molecular clouds near the SNR. This may be the case for TeV sources such as W44, W51C, IC 443 or W28.
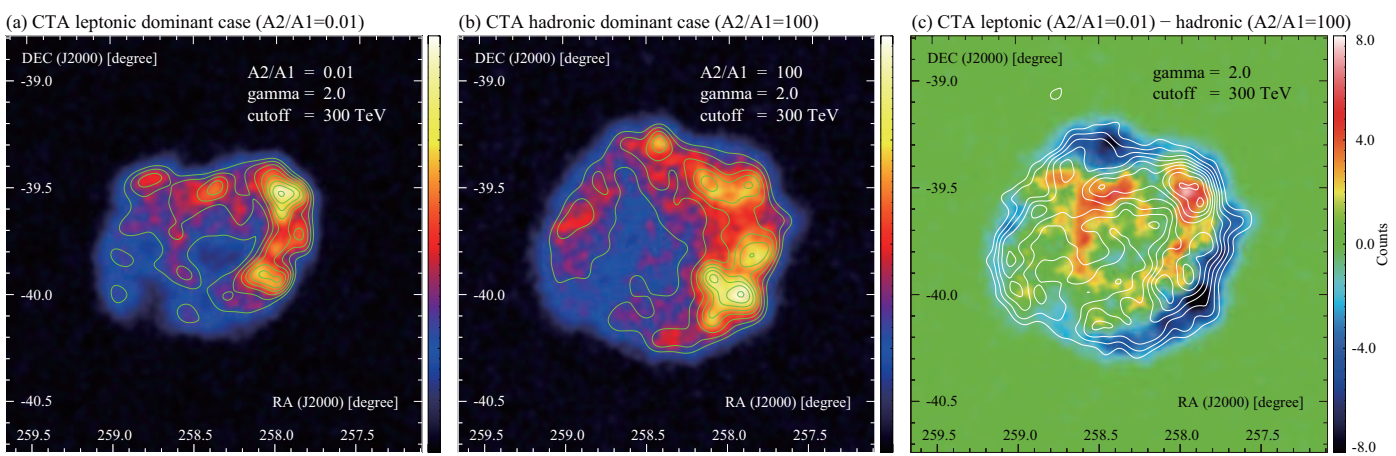

Figure 3: Simulated gamma-ray images of RXJ1713.7-3946 in different scenarios (a) lepton-dominated case and (b) hadron-dominated case. The green contours show (a) XMM-Newton X-ray intensity and (b) total interstellar proton column density [308], smoothed to match the PSF of CTA. The subtracted image of (a) - (b) is also shown in (c). The white contours correspond to the H.E.S.S. VHE gamma-ray emission. Find original figure and more details of the model parameters in [19].

CTA will offer an extended energy coverage going as low as $30 \mathrm{GeV}$ and as high as $300 \mathrm{TeV}$. Improved angular resolution will allow better morphological studies, which will enable to better correlate emission at $\mathrm{TeV}$ and lower frequencies. The expected significant increase of the number of TeV SNRs by CTA, especially during the galactic plane KSP, should allow to make population studies, a key issue to substantiate the SNR origin of galactic cosmic rays. A second KSP is fully focused on the study of PeVatrons. On one hand the KSP will be dedicated to observations of known sources with particularly hard spectra and with hints for a possible spectral extension into the multi-TeV energy domain. On the other hand a deep observation of the SNR RXJ1713.7-3946 will be performed. This is one of the best studied SNRs at gamma-ray energies and a considerable body of theoretical work has been devoted to it. Moreover, it is embedded in a dense, well-studied region that provides a large amount of target material. This is the optimal situation to investigate the interaction of cosmic rays, which are believed to escape from the acceleration region and collide 
with these clouds. Figure 3 illustrates how the CTA observation may allow to disentangle the leptonic and hadronic contributions based on their different morphologies.

The Galactic Center deserves special mention. VHE emission probably associated to the supermassive black hole has been reported by the current generation of IACTs, but H.E.S.S. has recently reported evidence[22] for the presence of protons with energies in excess of $1 \mathrm{PeV}$ in the central $10 \mathrm{pc}$ of our galaxy. These protons may originate at the accretion flow into the supermassive black hole or at its termination, which would establish the Galactic Center as a PeVatron. CTA will dedicate a sizable amount of observation time to this region as part of the galactic center KSP.

\subsubsection{Pulsar Wind Nebulae}

One of the surprises of the H.E.S.S. galactic plane survey was the discovery of a large population of VHE-emitting Pulsar Wind Nebulae (PWN). More than 30 PWN have been reported so far and PWN in fact dominate the list of galactic sources at this energy domain. Emission results from the population of electrons and positrons that leave the pulsar at the center of the PWN as a "cold wind" and are subsequently re-accelerated at the shock between this wind and the surrounding medium.
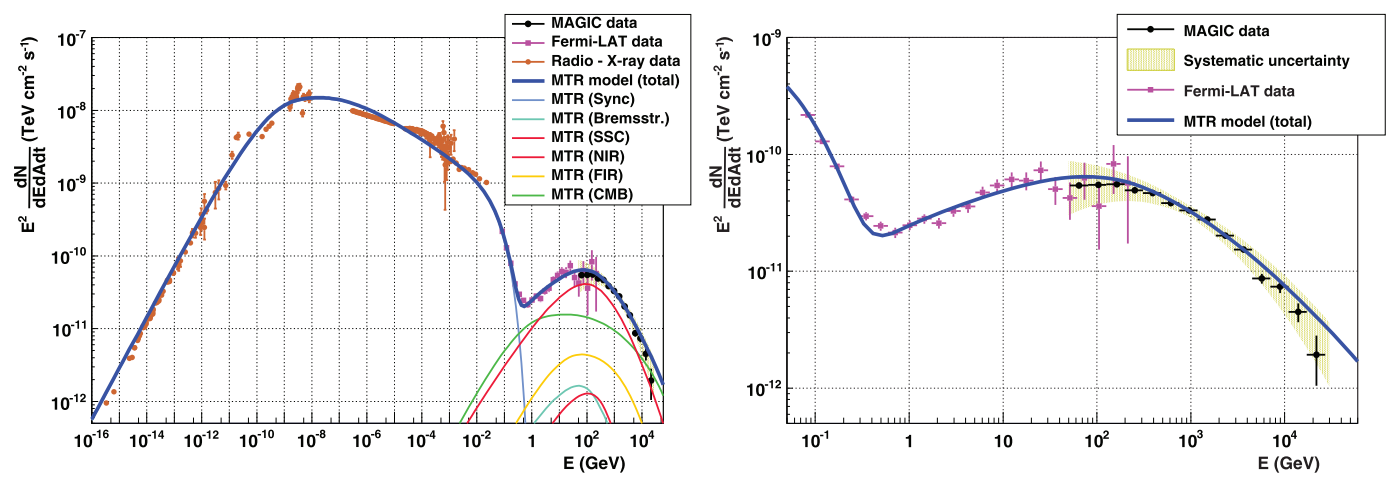

Figure 4: On the left: Overall SED of the Crab Nebula from radio to $\gamma$-rays. Lines are best fit results based on a leptonic model (MTR). The thin lines show individual components of the photon spectrum (see the inlay), and the thick blue line identifies the overall emission. On the right: Zoom in the gamma ray regime. For details of the model and the references to the data, the reader is referred to [23] where the original version of this plot was published.

The standard candle at VHE and best studied PWN at all wavelengths is the Crab Nebula. Figure 4 shows its overall spectral energy distribution (SED). PWN leptons emit at all energy domains from radio to VHE $\gamma$-rays. Up to tens of $\mathrm{GeV}$ the dominant process is synchrotron emission, whereas higher energy photons are produced through IC. The IC emission from the Crab Nebula was detected for the first time above $700 \mathrm{GeV}$ by the pioneering Whipple imaging atmospheric Cherenkov telescope in 1989. Since then, IACTs have successfully extended the Crab Nebula differential energy spectrum from few hundred $\mathrm{GeV}$ up to $80 \mathrm{TeV}$. However, the spectrum below 200 $\mathrm{GeV}$ has been observed only recently, revealing the long-anticipated IC peak in the distribution. At low energies, space-based instruments, like Fermi-LAT, have improved the sensitivity in the energy range between few and hundred GeV, with MAGIC finally overlapping with Fermi-LAT [23]. The 
population of IC target photons is not fully defined. No model fully accounts for all morphological and spectral features of this object.

The VHE part of the SED or IC peak contains information of the total energy released by the pulsar in its life time. PWN essentially act like calorimeters at VHE and observations allow to investigate their time-evolution. This also implies a better understanding of the still unknown mechanism of how the pulsar releases its rotational energy from the cold relativistic wind to a kinetic one with Lorenz factor of $3 \cdot 10^{6}$ at the termination shock.

VHE PWN are very extended (up to $1-2^{\circ}$ ) so their study will benefit from the wide field of view of CTA[24]. The large energy range covered by CTA will be crucial to understand cooling effects in the electron parent populations, including the resolution of internal structures and the possibility to disentangle between synchrotron or adiabatic losses. Due to its increase sensitivity observations with CTA will allow to increase the VHE population of these sources and, most importantly, to achieve an homogeneous sampling of the Galaxy.

\subsubsection{Pulsars}

Thanks to Fermi-LAT observations pulsars are now acknowledged as a distinct class of galactic $\gamma$-ray sources. Fermi-LAT Second Source Catalog contains nearly 90 individual pulsars including 27 firm detections of millisecond pulsars (MSPs). However little is known about the properties of pulsars above $10 \mathrm{GeV}$ where the performance of Fermi-LAT performance is limited. MAGIC discovered the first VHE pulsar when it detected pulsed emission the Crab. VERITAS has shown that emission extends up to $400 \mathrm{GeV}$ and MAGIC even reports pulsed emission beyond $1 \mathrm{TeV}$, as opposed to an exponential cutoff as might have been expected (see [24] and references within). HESS-II has reported pulsed emission from the Vela pulsar, but no indication of a VHE spectral extension yet. The emission mechanism behind the VHE tail in the Crab pulsar is unknown.

One of the main motivations of CTA is to explore the energy range of $10 \mathrm{GeV}$ to $100 \mathrm{GeV}$, by using very large reflectors to decrease the energy threshold to a few tens of GeV. CTA should thus offer a unique opportunity to explore the most extreme energetic processes in pulsar magnetospheres and beyond their light cylinders. High quality spectral, phase-resolved properties of the VHE radiation from Crab will be essential to help to develop realistic models of the magnetospheric gaps. Under the hypothesis of the existence of the VHE Crab-like energy tails, a large fraction (up to $40 \%$ ) of the brightest Fermi pulsars may be detected with CTA.

Globular clusters (GC) are known to contain many MSPs and they are expected to be the sources of unpulsed VHE radiation. The most attractive GC harboring probably as many as 200 MSPs (more than 20 radio-pulsars are known to date) is 47 Tucanae (47 Tuc). H.E.S.S. has set an Upper Limit (UL), which appears stringent enough to constrain the parameter space (number of MSPs vs. ambient magnetic field strength) in a simple model of this GC. H.E.S.S. has also reported on the discovery of the VHE radiation from vicinity of Terzan 5 (Ter 5). This radiation may be due to MSPs in the cluster, although a significant offset from the location of Ter 5 has no satisfactory explanation so far. For other globular clusters with lower content of MSPs the expected flux level between $100 \mathrm{GeV}$ and $10 \mathrm{TeV}$ is probably beyond the reach of present-day IACTs. However CTA may be sensitive enough to detect some globular clusters and even to resolve their spectral shapes at VHE. CTA may allow the detailed study of the major ingredients of globular clusters which link MSPs to the expected VHE emission. 


\subsubsection{Binaries}

By detecting emission from the binary systems PSR B1259-63, LS 5039, LS I +61 303 and HESS J0632+057 the current generation of IACTs has established a new population of VHE sources: gamma ray binaries, whose luminosity peaks at VHE [25]. This is evidence for very efficient particle acceleration in binary systems containing a compact object. A bright Fermi-LAT source, 1FGL J1018.6-5856, has been proposed to be a new gamma-ray binary that could be associated with a H.E.S.S. source.

All of these systems may host a pulsar and acceleration may take place at a shock between the pulsar and the companion star winds. Except for the hint of a VHE flare in the black hole binary Cyg X-1, there is no evidence for VHE emission stemming from microquasars even if emission at lower energy gamma- rays $(\mathrm{E}>100 \mathrm{MeV})$ has been reported for Cygnus X-3 and Cyg X-1.

CTA good sensitivity in the range $10-100 \mathrm{TeV}$ will allow us to probe the acceleration mechanism of gamma ray binaries, the strength of the magnetic field, intrinsic orbital/short timescale variability of the accelerator/emitter, and discriminate between lepton or hadron primaries. The detection of sporadic emission from microquasars will probably depend on external triggers or deep monitoring campaigns of specific objects like Cyg X-1, Cyg X-3 or GRS 1915+105. The high sensitivity and good angular resolution will allow also for imaging of possible extended emission at the termination of the generated outflows in systems such as Cyg X-1 or SS 433 (for more details of the capability of CTA for binary observations see [26]).

\subsubsection{Star clusters and star forming systems}

Cosmic rays may be a key regulator of star formation since they are able to penetrate to the innermost regions of molecular clouds, where they provide the only source of ionization and may thus regulate the conditions for star formation. If this is the case one would expect correlation between the $\gamma$-ray luminosity and the star formation rate.

Evidence for VHE emission in star forming systems has been found both inside and outside our galaxy. A wealth of sources has been detected in the Cygnus region by Fermi-LAT, HEGRA, Milagro, VERITAS and Argo-YBJ. Fermi-LAT may in fact have observed a cocoon of freshly accelerated cosmic rays. H.E.S.S. has found emission centered but extending over a region 20 times larger than the star cluster Westerlund 2. Beyond our galaxy the two starbursts M 82 and NGC 253 have been detected.

CTA intends to study the $\gamma$-ray luminosity vs star formation rate correlation at all scales, ranging from star forming regions in our galaxy (Carina, Cygnus and Westerlund 1) to local galaxies (M 31), starburst galaxies (M 82 and NGC 253) and Ultraluminous Infrared Galaxies (Arp 220).

\subsection{Extragalactic physics}

\subsubsection{Blazars}

Most of the extragalactic VHE sources[27, 28] are AGNs whose jet axes are almost aligned with our line of sight and hence show beaming effects. This is the so-called "blazar" class, which is made up of BL Lacs and Flat Spectrum Radio Quasars (FSRQs). Five FSRQ and more than 50 BL Lacs, mostly HBLs, have been discovered at VHE. 
The bolometric energy output of AGN jets is often dominated by the $\gamma$-ray range, so the $\gamma$-ray luminosity sets a relevant lower limit to the jet energetics once the bulk Lorentz factor is properly taken into account.

The location of the emission region is a matter of strong debate. Extremely fast variability, with time scales as short as a few minutes, has been observed in a few VHE AGNs (PKS 2155-304, Mrk 501, PKS 1222+21). This might imply that the emission region is located near the central black hole. However the extension of the spectrum of PKS 1222+21 up to hundreds of GeV is at odds with an emission region inside the Broad Line Region.

Because of its enhanced sensitivity and lower threshold energy, CTA is expected to increase the sample of AGN detected in the VHE range by about one order of magnitude. This is especially important for types of blazars for which only a few objects have been detected such as low-frequency peaked VHE AGNs or transition objects (e.g. BL Lac to FSRQ or HBL to LBL). The population of AGNs will also extend to higher redshifts, thus allowing to study their evolution with cosmic time.

CTA's higher sensitivity opens the possibility to monitor a larger number of AGNs as well and perform studies of their duty cycle and temporal characteristics.

\subsubsection{Extragalactic background light}

The extragalactic background light (EBL) is a fundamental observational quantity to understand the evolution of our Universe. All energy releases from resolved and unresolved extragalactic sources, and light from any truly diffuse background (excluding the cosmic microwave background), contribute to its intensity and spectral energy distribution. As such it is essential to test our knowledge of the formation and evolution of stellar objects and galaxies. It also sets limits on exotic energy releases in the universe.

VHE $\gamma$-rays are attenuated on their way to Earth by pair producing with photons of the EBL and $\mathrm{CMB}[29]$. The EBL affects the spectrum of the sources in the $10 \mathrm{GeV}$ to $10 \mathrm{TeV}$ energy regime. Knowledge of the EBL intensity and spectrum allows to reconstruct the intrinsic blazar spectrum in a crucial energy regime that can be used to test particle acceleration mechanisms. Conversely, knowledge of the intrinsic VHE spectrum and the detection of blazars enables to set strong limits on the EBL and its evolution.

The broad energy range covered by CTA includes energies where $\gamma$-rays are unaffected from absorption while propagating in the EBL, and extends to an energy regime where VHE spectra are strongly distorted. This will help to reduce systematic effects in the spectra from different instruments, leading to a more reliable EBL determination, and hence will make it possible to constrain blazar models up to the highest energies with less ambiguity. For more details of the potential of CTA for EBL studies see [30].

\subsubsection{Radiogalaxies}

The current generation of IACTs has established the existence of a new class of AGNs with VHE emission: radiogalaxies[28, 27]. In a way detecting only blazars at VHE energies provides biased information about AGN jets, because blazars are always observed at small viewing angles. Instead radiogalaxies display moderate or negligible Doppler boosting, so they are key to understand radio-loud AGNs. 
As of today only 5 radioagalaxies have been detected at VHE. CTA will boost the number of detections. Especially relevant may be its improved sensitivity to produce light curves which may be linked to light curves at other wavelengths and angular resolution to locate the VHE emission zones of nearby radiogalaxies.

\subsubsection{Clusters of galaxies}

We observe diffuse radio emission in clusters of galaxies coming from electrons and positrons either at their cores or at so-called radio relics. Whether they are directly accelerated or protons are accelerated and later produce them as secondaries is an open question. Protons shall produce $\gamma$-rays via $\pi^{0}$ decay, so such hadronic emission may be observable in the HE or VHE ranges. Searches with Fermi-LAT or MAGIC has shown no evidence for such emission and the corresponding ULs constrain the fraction of energy contained in cosmic rays below a few $\%$ of the thermal energy in the cluster. CTA is expected to improve this UL by a factor 5 with a dedicated KSP. If no diffuse VHE emission is observed we can establish a remarkably low constrain to the ratio of cosmic ray to thermal energies. Conversely the distribution of a putative diffuse emission would help to study how cosmic rays propagate in the cluster.

\subsubsection{Gamma Ray Bursts}

Gamma-ray bursts (GRB) are events, occurring at an average rate of a few per day throughout the universe, which for a period of seconds outshine all other sources of $\gamma$-rays in the sky. So-called long GRBs are associated to Supernovae. Fermi-LAT has recently shown that a substantial fraction of GRBs have photon spectra which extend at least to tens of GeV. This could be due to Inverse Compton emission or hadronic interactions[31].

No GRB has ever been detected with IACTs, even if a few of them have been observed relatively short after its prompt emission. With its low energy threshold, large effective area and rapid slewing capabilities, CTA may be able to measure the spectra and variability of GRBs at multi-GeV energies with unprecedented photon statistics. Light curves with much higher photon statistics than Fermi-LAT may be instrumental to understand the high energy $\gamma$-ray production mechanism. Detection of the prompt emission may me challenging even if LSTs are designed to re-point to any point of the sky in less than 20 seconds. conditions are probably more favorable to detect the GRB afterglow. See [32] for more details of the capabilities to CTA to study GRBs.

\section{References}

[1] W. B. Atwood et al., The Large Area Telescope on the Fermi Gamma-Ray Space Telescope Mission, ApJ 697 (2009) 697, 1071.

[2] J. Aleksić et al., The major upgrade of the MAGIC telescopes, Part I: The hardware improvements and the commissioning of the system, submitted to Astroparticle Physics and arXiv:1409.6073.

[3] J. Aleksić et al., The major upgrade of the MAGIC telescopes, Part II: The achieved physics performance using the Crab Nebula observations, submitted to Astroparticle Physics and arXiv:1409.5594.

[4] F. A. Aharonian et al., Observations of the Crab nebula with HESS. Astronomy \& Astrophysics 457 (2006) 899-915. 
[5] J. Holder et al., Dec. 2008. Status of the VERITAS Observatory. In: F. A. Aharonian, W. Hofmann, F. Rieger (Eds.), American Institute of Physics Conference Series. Vol. 1085 of American Institute of Physics Conference Series. pp. 657-660.

[6] A. M. Hillas, Evolution of ground-based gamma-ray astronomy from the early days to the Cherenkov Telescope Arrays, Astrop. Phys. 43 (2013) 19-43.

[7] R. Atkins et al., TeV gamma-ray survey of the northern hemisphere sky using the Milagro observatory, ApJ 608 (2004) 680-685

[8] M. Amenomori et al., A northern sky survey for steady tera-electron volt gamma-ray point sources using the Tibet Air Shower Array, ApJ 633 (2005) 1005-1012.

[9] A. Sandoval for the HAWC collaboration, High Energy Astrophysics with the HAWC Gamma Ray Observatory, proceedings of RICAP-14, Noto (Sicily, Italy), September 30th- October 3rd, 2014, available at https://agenda.infn.it/conferenceDisplay.py?confId=7620.

[10] B.S. Acharya et al., Introducing the CTA concept, Astrop. Phys. 43 (2013) 3.

[11] J. Cortina and M. Teshima for the CTA Consortium (2015) in proc. of the 34th International Cosmic Ray Conference (ICRC); ibid arXiv:1508.06438.

[12] M. Garczarczyk et al., the MST for the CTA Consortia (2015) in proc. of the 34th ICRC; ibid arXiv:1508.01361.

[13] K. Byrum et al for the CTA Consortium (2015) in proc. of the 34th ICRC; ibid arXiv:1509.03074.

[14] J-F. Glicenstein et al. for the CTA Consortium (2015) in proc. of the 34th ICRC; ibid arXiv:1508.1508.06555.

[15] G. Puehlhofer et al. for the CTA Consortium (2015) in proc. of the 34th ICRC; ibid arXiv:1509.02434.

[16] T. Montaruli, G. Pareschi and T. Greenshaw for the CTA Consortium and the SST-1M, ASTRI and GCT sub-consortia (2015) in proc. of the 34th ICRC, ibid arXiv:1508.06472.

[17] S. Vercellone for the ASTRI and CTA consortia, Status of the ASTRI, these proceedings.

[18] Special issue of Astroparticle Physics: Volume 43 (March 2013): "Seeing the High-Energy Universe with the Cherenkov Telescope Array - The Science Explored with the CTA".

[19] CTA Construction Project Science Technical Design Report, OBS-TDR/141106.

[20] A.R. Bell, Cosmic ray acceleration, Astrop. Phys. 43 (2013) 56.

[21] F. A. Aharonian, Gamma rays from supernova remnants, Astrop. Phys. 43 (2013) 71.

[22] F. A. Aharonian et al., Acceleration of petaelectronvolt protons in the Galactic Centre, Nature 531 (2016) 476.

[23] J. Aleksić et al., Journal of High Energy Astrophysics 5-6 (2015) 30-38.

[24] E. de Oña-Wilhelmi et al., Prospects for observations of pulsars and pulsar wind nebulae with CTA, Astrop. Phys. 43 (2013) 287-300.

[25] W. Bednarek, High energy $\gamma$-ray emission from compact galactic sources in the context of observations with the next generation Cherenkov Telescope Arrays, Astrop. Phys. 43 (2013) 81.

[26] J. M. Paredes et al., Binaries with the eyes of CTA, Astrop. Phys. 43 (2013) 301.

[27] A. Reimer and M. BÃúttcher, Studies of active galactic nuclei with CTA, Astrop. Phys. 43 (2013) 103. 
[28] H. Sol et al., Active Galactic Nuclei under the scrutiny of CTA, Astrop. Phys. 43 (2013) 215.

[29] E. Dwek and F. Krennrich, The extragalactic background light and the gamma-ray opacity of the universe, Astrop. Phys. 43 (2013) 112.

[30] D. Mazin et al., Potential of EBL and cosmology studies with the Cherenkov Telescope Array, Astrop. Phys. 43 (2013) 241.

[31] P. Meszaros, Gamma ray bursts, Astrop. Phys. 43 (2013) 134.

[32] S. Inoue et al., Gamma-ray burst science in the era of the Cherenkov Telescope ArrayOriginal Research Article Astrop. Phys. 43 (2013) 252. 